\title{
Use of high dose corticosteroids in patients with inoperable brain tumours
}

\author{
A B R A A M L I B ERMAN, Y VES LE BR UN, PETER GLASS, \\ ALBERT GOODGOLD, WARREN LUX, ARLENE WISE, \\ A N D J O S P H R A N S O H F F \\ From the Department of Neurology and the Department of Neurosurgery, New York University \\ Medical Center, New York USA
}

SUMMARY Eleven patients with inoperable brain tumours were treated with high doses of corticosteroids (methylprednisolone $200-2000 \mathrm{mg}$ /day) for up to 151 days (mean 55 days). Neurological improvement occurred in eight patients on high doses after deterioration on conventional doses (methylprednisolone $80-120 \mathrm{mg} /$ day). In two patients steroids could be completely discontinued for several months. Serious adverse effects included sepsis in three patients and myopathy in two. All three patients with sepsis also received chemotherapy. There were no deaths that could be attributed to steroids. The most likely effect of high dosage steroids is reduction of cerebral oedema. It is conceivable that in some instances high dose steroids may also result in tumour inhibition or oncolysis or both.

Corticosteroids are valuable adjuncts in the management of patients with brain tumours. In the doses conventionally used $(80-120 \mathrm{mg} /$ day of methylprednisolone, 16-24 mg/day of dexamethasone) their principal action is reduction of peritumoural oedema. While all patients eventually show signs of neurological deterioration it was rare, until recently, to exceed $120 \mathrm{mg} /$ day of methylprednisolone. The rationale for not exceeding that dose is unclear but is probably based on fear of adverse effects: sepsis, gastrointestinal haemorrhage. Recently high dosage of steroids has been used successfully to treat patients with brain tumours. We review our experience with high dose steroids (200 to $2000 \mathrm{mg}$ /day of methylprednisolone) because the published reports are few and information on adverse effects limited (Renaudin et al., 1973; Marshall et al., 1975). We would also like to comment on the possibility of tumour inhibition and/or oncolysis with high dose steroids.

\section{Methods}

In a one year period 11 patients with brain tumours, representing $15 \%$ of patients with brain

Supported in part by The National Cancer Institute, Contract No. NO1-CM-71174.

Address for reprint requests: Dr A. N. Lieberman, Department of Neurology, New York University Medical Center, 550 First Avenue, New York, NY 10016, USA.

Accepted 15 February 1977 tumours (both primary and secondary) at the New York University Medical Center, received $200 \mathrm{mg}$ per day (or more) of methylprednisolone for at least 21 consecutive days. Methylprednisolone was administered in increasingly high doses to control increased intracranial pressure. Six patients had primary and five had metastatic tumours. Among the latter, two were from the lung, one was from the colon, and in two no primary source could be found. Ages ranged from 31 to 74 years (mean 57). All patients on admission were symptomatic, and in all the location of the tumour precluded surgery: dominant hemisphere in six, multiple in three, and deep thalamic in two. Five patients had received previous treatment. This consisted of radiation in four patients and surgery and radiation (6000 rads) in one patient who had originally presented with a nondominant hemisphere astrocytoma and at a later date with a recurrent inoperable dominant hemisphere astrocytoma. Seven patients received radiation while on high dose steroids. Six had not previously received radiation while one had received less than a full course. Six patients received concurrent chemotherapy.

Among the 11 patients on more than $200 \mathrm{mg} /$ day of methylprednisolone there was a subgroup of six who received $500 \mathrm{mg} /$ day for up to 35 days (mean 13 days), and within this subgroup there were five patients who received $1000 \mathrm{mg} /$ day for 
up to 21 days (mean six days). Three of the latter received up to $2000 \mathrm{mg} /$ day. Eight of the patients had been started on conventional doses of methylprednisolone, and this was increased because of neurological deterioration. In three patients steroids were begun in high dosage at the onset because of signs of uncal herniation with rostral-caudal deterioration. The clinical course of the patients was followed by a graded neurological examination, isotopic brain scan, and computerised tomography. Nine of the 11 patients died, and postmortem examinations were performed in five.

\section{Results}

The effects of treatment are summarised in the Table. Improvement on high dose steroids occurred in all of the eight patients who had shown deterioration on conventional doses and continued up to $1000 \mathrm{mg} /$ day. No additional improvement occurred beyond this dose up to $2000 \mathrm{mg}$ /day. In one typical example a patient with a dominant hemisphere tumour one year after radiation had become aphasic and unable to walk. She was placed on methylprednisolone in gradually increasing doses. Resolution of symptoms occurred on $120 \mathrm{mg} / \mathrm{day}$, and she remained asymptomatic for several weeks. She again became aphasic and unable to walk. Methylprednisolone was increased to $500 \mathrm{mg} /$ day and she regained her ability to speak and walk. Improvement lasted for six weeks but was followed by neurological deterioration and death.

In two patients with primary brain tumours steroids could be completely discontinued for several months. Both patients had dominant hemisphere tumours and had received radiation and chemotherapy. Both had been treated with $80 \mathrm{mg} /$ day of methylprednisolone and had become steroid dependent, attempts at decreasing the dose of methylprednisolone resulting in neurological deterioration. When deterioration began to occur despite $80 \mathrm{mg} /$ day of methylprednisolone the dose was rapidly increased to $500 \mathrm{mg} /$ day and maintained at this level for 21 days. In both patients there was prompt improvement and steroids could be discontinued completely. One patient continues to maintain his improvement 14 months later. He has a mild expressive aphasia and right hemiparesis. He lives at home, is independent in activities of daily living, and regularly comes to the hospital for examinations. In the other patient symptoms recurred after three months and progressed rapidly till death.

Dramatic improvement on high dose methyl- prednisolone occurred in two of three patients with uncal herniation and signs of rostral-caudal deterioration who had not been previously treated with steroids. One patient with metastases from an undifferentiated lung carcinoma was decerebrate on admission. He was started on methylprednisolone $1000 \mathrm{mg} /$ day and four days later regained consciousness and the ability to walk. At this point, radiation therapy was begun. Over the next 12 days methylprednisolone was decreased to 500 $\mathrm{mg} /$ day. This resulted in rapid neurological deterioration which was not reversed with $2000 \mathrm{mg}$ / day of methylprednisolone.

Three patients $(28 \%)$ developed serious bacterial infections while on steroids and chemotherapy (methyl-1-(2-chloroethyl)3-(4 methylcyclohexyl)-1nitrosourea; Semustine). One patient, a diabetic man, developed four separate major infections all of which were treated successfully. At a later date he developed a severe myopathy and became unable to walk. Another patient developed disseminated herpes zoster and at a later date a severe myopathy. While it is difficult to state whether the steroids, the chemotherapy, or the combination of both were responsible for the decreased resistance leading to infection, the morbidity of $28 \%$ compares unfavourably with our morbidity of $5 \%$ in patients on chemotherapy and conventional doses of methylprednisolone. There were no deaths that could be attributed to steroids. There were several minor adverse effects. All 11 patients developed features of hyperadrenocorticalism; six developed a monilial pharyngitis (responsive to nystatin); five had persistently elevated blood sugar levels but only the known diabetic required insulin. There were no episodes of gastrointestinal bleeding. Two patients, both with metastatic tumours, developed multiple pulmonary emboli requiring anticoagulant treatment. In both, the emboli were considered to be unrelated to the steroids.

\section{Discussion}

Our data indicate that high doses of corticosteroids are an effective and relatively safe shortterm treatment for patients with brain tumours who are no longer responding to conventional doses. The data imply that an arbitrary upper limit on dose should not be set but that steroids should be increased as necessary in each patient. They also indicate that there is a limit to the effectiveness of steroids as with dosage above $1000 \mathrm{mg} /$ day of methylprednisolone we observed no additional improvement. The high incidence noted of serious but treatable infections and of 





myopathy is disconcerting but, given the desperate condition of our patients, these effects were thought to be justifiable. The sustained improvement noted in two patients after treatment with high dose steroids raises the possibility that steroids may have an inhibitory and/or oncolytic effect on the tumour.

Corticosteroids were introduced as adjuncts in the management of brain tumours in the early 1950 s to prevent postoperative adrenocortical collapse in patients undergoing surgery for sellar and parasellar tumours (Matson and McLaurin, 1952; Tytus et al., 1955). At this time steroids were also used to suppress endogenous adrenal secretions in patients with endocrine sensitive breast tumours. While the results of such treatment were disappointing some of the earlier patients also had extensive cerebral metastases. The dramatic improvement in neurological symptoms in these patients, and the unusually smooth postoperative course in the steroid treated patients with sellar and parasellar tumours, led to the use of steroids in patients with other brain tumours (Kofman et al., 1957; Galicich et al., 1961; Ruderman and Hall, 1965).

These authors reported that steroids are effective in reducing the neurological symptoms in more than $70 \%$ of patients with brain tumours, both primary and metastatic. Onset of action is rapid, within 24 to 48 hours, but is usually shortlived (three to six months). Response is independent of the type of steroid (hydrocortisone, cortisone, prednisone, methylprednisolone, dexamethasone) provided equivalent doses are used. The rapid onset of neurological improvement, the similarity of improvement in patients with different histological types of tumours, the similarity of improvement in a variety of diverse conditions having as their only common denominator cerebral oedema, and the failure to note regression of any primary extracerebral tumour has led most investigators to postulate that improvement is secondary to a reduction in oedema (Lippert et al., 1960; Beinderman et al., 1962; Rasmussen and Gulati, 1962; Harris, 1967; Long et al., 1972; Weinstein et al., 1973).

The way in which steroids alter oedema is not clear. The basic mechanism responsible for the development of cerebral oedema is an increase in cerebrovascular permeability to protein, water, sodium, and potassium (Klatzo, 1972) which is probably secondary to a disruption of the capillary endothelial junction and/or endothelial-glial junction (Harris, 1967; Long et al., 1972). Associated with the increased permeability, there is a change in the activity of certain hydrolytic enzymes that are normally bound to lysosomes (Bingham et al., 1971) and whose release further increases cerebrovascular permeability. There is also absence of a $\mathrm{Na}-\mathrm{K}$ stimulated adenosine-triphosphatase (Na-KATPase) in some oedematous structures (Krigman and Hurt, 1970). Na-K-ATPase functions as a compensatory mechanism for maintaining ionic integrity in the face of abnormal vascular permeability. Steroids may alter cerebral oedema by decreasing permeability through stabilisation of the capillary endothelial junction and/or endothelial-glial junction (Long et al., 1972); they may affect ionic transport across the capillary-glial interface, or they may prevent the release of disruptive lysosomal enzymes (Bingham et al., 1971). All these mechanisms involve changes in membrane structure and differ from the anti-oedema effect of dehydrating agents and diuretics. It is conceivable that a comparable change in membrane structure may also be involved in tumour inhibition and/or oncolysis in primary brain tumours. Thus both morphological abnormalities and reduced growth rates have been noted in tissue cultures of biopsied human central nervous system tumours at steroid concentrations of greater than $100 \mathrm{micrograms} / \mathrm{ml}$. However, complete lysis was seldom seen even with concentrations as high as $5000 \mathrm{micrograms} / \mathrm{ml}$ (Burton et al., 1967; Mealy et al., 1971; Wilson et al., 1972; Kajikama et al., 1974). Additional evidence for steroid induced tumour inhibition or oncolysis or both comes from in vivo studies where, in mice, steroids have been shown to have an inhibitory effect on transplanted melanomas (Kotsilimbas et al., 1967), transplanted lymphoid tumours (Baserga and Shubik, 1954), and a naturally occurring intracerebral ependymoblastoma (Shapiro and Posner, 1974). A decrease in the incorporation of tritiated thymidine into tumour DNA was also noted in the steroid treated mice with ependymoblastomas. Additionally, stcroids have been shown to have an inhibitory effect on a methylnitrosourea induced intracerebral glioma in rats (Gurcay et al., 1971), and in two different subcutaneously transplanted gliomas in mice and rats (Wright et al., 1969). The inhibitory effect of steroids on the growth of tumour cells in tissue culture has been attributed to an increase in the cell cycle time (tumour inhibition) rather than to cell death (oncolysis) (Wilson et al., 1972). The sustained improvement noted in two of our patients may represent such inhibition or oncolysis or both. So suggestive is the evidence for tumour inhibition and/or oncolysis that the Brain Tumor Study Group of the United States National Cancer Institute has undertaken a large multi-institutional cooperative study to in- 
vestigate this point and within the next few years a definitive answer may be forthcoming.

We wish to thank Dr. Irwin Feigin for his cooperation. We also wish to thank Ms. Kathleen Faridazar for typing the manuscript.

\section{References}

Baserga, R., and Shubik, P. (1954). The action of cortisone on transplanted and induced tumors in mice. Cancer Research, 14, 12-16.

Beinderman, E. F., Graf, C. J., and Fitzpatrick, T. (1962). Basic studies in cerebral edema: its control by a corticosteroid (Solumedrol). Journal of Neurosurgery, 19, 319-324.

Bingham, W. G., Paul, S. E., and Sastry, K. S. S. (1971). Effect of steroid on enzyme response to cold injury in rat brain. Neurology (Minneapolis), 21, 111-121.

Burton, A. F., Storr, J. M., and Dunn, W. L. (1967). Cytolytic action of corticosteroids on thymus and lymphoma cells in vitro. Canadian Journal of Biochemistry, 45, 289-297.

Galicich, J. H., French, L. A., and Melby, J. C. (1961). Use of dexamethasone in treatment of cerebral edema associated with brain tumors. Journal-Lancet (Minneapolis), 81, 46-53.

Gurcay, O., Wilson, C. B., and Barker, M. (1971). Corticosteroid effect of transplantable rat glioma. Archives of Neurology (Chicago), 24, 266-269.

Harris, A. B. (1967). Steroids and blood brain barrier alterations in sodium acetrizoate injury. Archives of Neurology (Chicago), 17, 282-297.

Kajikama, H., Harada, K., and Iguchi, T. (1974), Evaluation of anti-tumor effect of corticosteroid on human glioma in vitro. Hiroshima Journal of Medical Science, 23, 33-40.

Klatzo, I. (1972). Pathophysiological aspects of brain edema. In Steroids and Brain Edema, pp. 1-8. Edited by H. J. Neulen and K. Schurmann. Springer Verlag: Berlin, Heidelberg, New York.

Kofman, S., Garvin, J. S., and Nagamani, D. (1957). Treatment of cerebral metastases from breast carcinoma with prednisolone. Journal of the American Medical A ssociation, 163, 1473-1476.

Kotsilimbas, D. G., Meyer, L., and Berson, B. A. (1967). Corticosteroid effect on intracerebral melanomata and associated cerebral edema, some unexpected findings. Neurology (Minneapolis), 17, 223-226.

Krigman, M. R., and Hurt, J. P. (1970). Na-K-ATPase activity in focal cerebral edema associated with an experimental glioma in guinea pigs. Journal of
Neuropathology and Experimental Neurology, 29, 285-295.

Lippert, R. G., Svien, E. J., and Grindlay, J. H. (1960). The effect of cortisone on experimental cerebral edema. Journal of Neurosurgery, 17, 583589.

Long, D. M., Maxwell, R. E., and French, L. A. (1972). The effects of glucosteroids upon experimental brain edema. In Steroids and Brain Edema, pp. 65-76. Edited by H. J. Neulen and K. Schurmann. Springer Verlag: Berlin, Heidelberg, New York.

Marshall, L. F., King, J., and Langfitt, T. W. (1975). The complications of high dose corticosteroid therapy in neurosurgical patients: a prospective study. Presented at the American Association of Neurological Surgeons, Miami, Florida.

Matson, D. D., and McLaurin, R. L. (1952). Cortisone and ACTH as an adjunct to the surgery of craniopharyngiomas. New England Journal of Medicine, 246, 568-571.

Mealy, J., Chen, T. T., and Schanz G P (1971).. Effects of dexamethasone and methylprednisolone on cell cultures of human glioblastomas. Journal of Neurosurgery, 34, 324-334.

Rasmussen, T., and Gulati, D. R. (1962). Cortisone in the treatment of post-operative cerebral edema. Journal of Neurosurgery, 19, 535-544.

Renaudin, J., Feiver, D., and Wilson, C. B. (1973). Dose dependency of decadron in patients with partially excised brain tumors. Journal of Neurosurgery, 39, 302-305.

Ruderman, N. B., and Hall, T. C. (1965). Use of glucocorticoids in the palliative treatment of metastatic brain tumors. Cancer, 18, 298-306.

Shapiro, W. R., and Posner, J. B. (1974). Corticosteroid hormones: effects in an experimental brain tumor. Archives of Neurology (Chicago), 30, 217221.

Tytus, J. S., Holbrooke, S. S., and Kahn, E. A. (1955). Cortisone as an aid in the surgical treatment of craniopharyngiomas. Journal of Neurosurgery, 12, 555-564.

Weinstein, J. D., Toy, F. J., and Jaffe, M. E. (1973). The effect of dexamethasone on brain edema in patients with metastatic brain tumors. Neurology (Minneapolis), 23, 121-129.

Wilson, C. B., Barker, M., and Hoshino, T. (1972). Steroid induced inhibition of growth in glial tumors: a kinetic analysis. In Steroids and Brain Edema, pp. 95-100. Edited by H. J. Neulen and K. Schurmann. Springer Verlag: Berlin, Heidelberg, New York.

Wright, R. L., Shaumba, B., and Keller, J. (1969). The effect of glucocorticosteroids on growth and metabolism of experimental glial tumors. Journal of Neurosurgery, 30, 140-145. 